\title{
Laminite crônica em equídeos da raça Crioula: características clínicas e radiográficas
}

\author{
Chronic laminitis in Criollo horses: clinical and radiographic characteristics
}

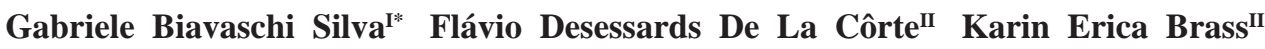 \\ Sergio da Silva Fialho ${ }^{\text {II }}$ Roberta Carneiro da Fontoura Pereira ${ }^{\mathrm{I}}$
}

RESUMO

Este estudo visou analisar o perfil clínico $e$ radiológico de cavalos crioulos com laminite crônica internados na Clínica de Equinos no período de 2010 a 2011. A partir das fichas de registro, avaliações clínicas diárias e estudos radiográficos, foram computados nove equinos, cinco fêmeas e quatro machos,

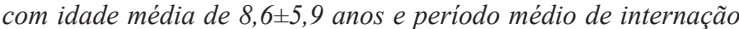
de 104,2 $\pm 91,7$ dias. As fêmeas representaram 55,6\% (5/9) da casuística, enquanto os machos inteiros representaram 44,4\% (4/9); 22,2\% (2/9) dos animais apresentaram laminite nos quatro membros, enquanto $77,8 \%$ (7/9) apresentaram laminite apenas nos membros anteriores. Quanto às causas da laminite, 11,1\% foram decorrentes de endotoxemia (1/9), 44,4\% (4/9) de sindrome metabólica equina e, em 44,4\% (4/9), não foi possível determinar a origem. Afundamento $\geq 20 \mathrm{~mm}$ da terceira falange ocorreu em $66,7 \%$ (6/9) dos animais. Apenas 11,1\% (1/9) dos animais apresentaram afundamento distal unilateral. Não houve correlação entre o afundamento da coluna óssea e o grau de claudicação. Dos nove animais deste estudo, 66,7\% (6/9) apresentaram rotação $\geq 5.5^{\circ} \mathrm{em}$ pelo menos um dos membros. Quanto maior o grau de rotação, maior o grau de claudicação $(0.1 \leq r<0.5 ; P<0.05)$. Dois pacientes $(22,2 \%)$ foram submetidos à tenotomia do flexor digital profundo. A resistência à insulina foi a causa predisponente a laminite mais comum neste estudo. As terapias de suporte, cuidados intensivos de enfermagem, casqueamento e ferrageamento corretivos resultaram em alta taxa de sobrevivência, permitindo que, após a alta, os animais pudessem ser mantidos com conforto sem uso de medicação.

Palavras-chave: laminite crônica, equinos, rotação, afundamento, síndrome metabólica.

\section{ABSTRACT}

This study aimed to analyze the clinical and radiographic profile of Criollo horses affected by chronic laminitis brought to the Equine Clinic between 2010 and 2011. Medical records, daily clinical evaluations and radiographic studies were analyzed. Data of nine horses, 55.6\% (5/9) females and 44.4\% (4/9) intact males with a mean age 8.6 \pm 5.9 years and 104.2 \pm 91.7 days of hospitalization were included. Laminitis occurred on all four limbs on $22.2 \%$ (2/9), while $77.8 \%$ (7/9) occurred only on the forelimbs. One out of nine cases laminites was due to endotoxemia (11.1\%), 44.4\% (4/9) was related to equine metabolic syndrome and on $44.4 \%$ (4/9) the cause could not be determined. Sinking of third phalanx $\geq 20 \mathrm{~mm}$ identified on radiographic studies occurred on $66.7 \%$ (6/9) horses. Only $11.1 \%(1 / 9)$ of the animals presented unilateral sinking of the third phalanx. Sinking of the third phalanx was not correlated with the degree of lameness. Rotation of the distal phalanx $\left(\geq 5.5^{\circ}\right)$ on at least one member was observed in $66.7 \%(6 / 9)$. There was a positive correlation of the third phalanx rotation degree and the lameness degree $(0.1 \leq r<0.5 ; P<0.05)$. Two patients $(22.2 \%)$ required tenotomy of the deep digital flexor tendon. Insulin resistance was the predisposing cause laminitis most common in this study. Supportive therapy, intensive nursing care, corrective trimming and shoeing provided a high survival rate after discharge so that the animals could be kept comfortable without pain medication.

Key words: chronic laminitis, horses, rotation, sinking, equine metabolic syndrome.

\section{INTRODUÇÃO}

A laminite em equinos é uma doença grave e comum, sendo reconhecida como uma das mais importantes síndromes clínicas ao longo da história (HUNT \& WHARTON, 2010). A laminite não é uma doença primária, usualmente ocorrendo como sequela de quatro diferentes entidades clínicas: doenças associadas à sepse/endotoxemia; excesso

IPrograma de Pós-graduação em Medicina Veterinária, Universidade Federal de Santa Maria (UFSM), Av. Roraima, 1000, prédio 97, Camobi, 97105-900, Santa Maria, RS, Brasil. E-mail: gabibiavaschi@hotmail.com.br. *Autor para correspondência.

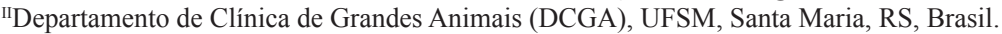


de peso apoiado sobre um membro devido à lesão no membro contralateral; síndrome de Cushing em cavalos velhos e síndrome metabólica equina (SME) (BELKNAP \& PARKS, 2011). POLLITT (2003) associou laminite com claudicação aguda de grau variável, envolvendo um ou mais membros, sendo que os membros anteriores são os mais acometidos.

O manejo da dor em cavalos com laminite é de extrema importância, pois alguns cavalos apresentam dor crônica no casco, que pode resultar em decúbito e, eventualmente, na necessidade de eutanásia por razões de bem-estar (SWANSON, 1999; HUNT \& WHARTON, 2010).

A laminite é considerada crônica a partir de 72 horas ou a partir dos primeiros sinais de deslocamento da terceira falange em relação à parede do casco, observados nas radiografias (HOOD, 1999). Cavalos com laminite crônica sofrem de deformação do casco e claudicação variável (BELKNAP \& PARKS, 2011).

Este estudo retrospectivo visou analisar parâmetros clínicos obtidos durante o exame físico além do grau de rotação e afundamento da terceira falange em cavalos Crioulos com laminite crônica, internados na Clínica de Equinos do Hospital Veterinário Universitário (HVU) da Universidade Federal de Santa Maria (UFSM), no período entre março de 2010 e outubro de 2011, a fim de identificar variáveis que possam ser usadas na indicação do tratamento mais adequado.

\section{MATERIAL E MÉTODOS}

O estudo retrospectivo foi realizado utilizando as informações das fichas de registro e tratamento que continham informações das avaliações clínicas diárias de nove cavalos Crioulos, internados com laminite crônica na Clínica de Equinos do HVU - UFSM, no período de março de 2010 a outubro de 2011. No momento da internação, os animais foram submetidos a um exame físico, acompanhado de exames complementares, como exame radiológico da falange distal e exames hematológicos para confirmar o diagnóstico e guiar a escolha do antiinflamatório não esteroidal (AINE) a ser utilizado no tratamento. No exame hematológico, avaliaramse hemácias, hemoglobina, hematócrito, volume corpuscular médio, concentração de hemoglobina corpuscular média, leucograma e fibrinogênio. No exame bioquímico, foram avaliados os níveis séricos de albumina, gamaglutamiltransferase, proteínas totais, aspartato aminotransferase, creatino quinase, fosfatase alcalina, creatinina e ureia.

Em casos de suspeita de síndrome metabólica, era realizado o teste de tolerância a glicose e insulina (TTGI), que era realizado a partir do momento em que os animais não apresentavam mais dor aguda, com frequência cardíaca abaixo de 48 batimentos por minuto, já que esta pode causar resistência à insulina e, dessa forma, alterar a interpretação dos resultados. O TTGI foi realizado de acordo com EILER et al. (2005). Nesse momento, também eram determinados o peso e a altura dos animais, com auxílio de fita e hipômetro, respectivamente.

Os critérios para inclusão no estudo foram: (1) cavalos da raça Crioula com laminite crônica; (2) existência de informações completas relativas ao exame clínico, tratamento e disponibilidade de exames radiográficos. A claudicação foi avaliada segundo OBEL (1948) em grau 1: não havia claudicação evidente ao passo, mas o cavalo trocava de apoio frequentemente em intervalos de poucos segundos; grau 2: o cavalo se movimentava voluntariamente, mas a marcha era forçada e era possível erguer um membro sem maiores dificuldades; grau 3: o cavalo se movia com relutância e resistia vigorosamente às tentativas de erguer o membro contra-lateral; e grau 4: o cavalo recusava a mover-se e só o fazia quando era forçado.

As imagens radiográficas utilizadas neste estudo foram obtidas usando um emissor portátil (BOWIE ${ }^{\circledR}$ ULTRALITE $10040 \mathrm{HF}$, EUA) e películas radiográficas (KODAK, T-MAT G/RA, EUA) de 25,4x20,3cm em chassis rígidos (ORTHO REGULAR CURIX screens, EUA). Para a projeção lateromedial, dois marcadores eram fixados no casco, um no ápice da ranilha, para avaliar a relação entre a terceira falange e a sola e outro na parede dorsal do casco para a avaliação de possível afundamento da coluna óssea e rotação da terceira falange. Na projeção dorso palmar/plantar, os cascos eram colocados sobre bloco de madeira sem marcadores para avaliar a presença de afundamento unilateral da terceira falange e equilíbrio do casco. O acompanhamento radiológico (projeção lateromedial e dorso palmar/ plantar) era realizado a cada 15 dias. As radiografias obtidas foram digitalizadas e mensuradas (grau de afundamento e rotação da terceira falange) através do software Metron-Hoof-Pro 5.19.

O grau de rotação da terceira falange foi determinado através de mensuração do ângulo formado entre a parede dorsal do casco e a parede dorsal da terceira falange, de acordo com BELKNAP 
\& PARKS (2011). Já o afundamento da coluna óssea foi avaliado por meio de mensuração da distância entre o limite proximal da parede dorsal do casco (identificado com um marcador de metal) e o limite proximal do processo extensor da falange distal (CRIPPS \& EUSTACE,1999b).

Os AINEs mais utilizados foram a fenilbutazona $\left(2,2 \mathrm{mg} \cdot \mathrm{kg}^{-1}\right.$ ou $4,4 \mathrm{mg} \cdot \mathrm{kg}^{-1}, 2$ xdia, via oral), cetoprofeno (2,2mg. $\mathrm{kg}^{-1}$ ou $3,4 \mathrm{mg} \cdot \mathrm{kg}^{-1}$, $2 x d i a$, via oral $)$, meloxican $\left(0,6 \mathrm{mg} \mathrm{kg}^{-1}, 1 \times\right.$ dia, via oral) e flunixin meglumine $\left(1,1 \mathrm{mg} \cdot \mathrm{kg}^{-1}, 1 \mathrm{xdia}\right.$, via intravenosa). Durante todo o período de tratamento com AINEs, os animais também receberam $1 \mathrm{mg} \cdot \mathrm{kg}^{-1}$, 1xdia, de omeprazole, por via oral.

Assim que chegavam ou enquanto houvesse presença de pulso digital positivo e lesões ulcerativas nos cascos, os animais eram mantidos com palmilhas de etil vinil acetato (EVA) fixadas com fita adesiva prateada, recobrindo a superfície solar. Após a redução do pulso digital, os animais eram submetidos a casqueamento/ferrageamento corretivo periódico mensal, de acordo com o controle radiológico da terceira falange.

A análise dos resultados obtidos foi realizada pelo método de correlação de Pearson.

\section{RESULTADOS E DISCUSSÃO}

Dos 13 casos de laminite avaliados durante o período de março de 2010 e outubro de 2011, nove cavalos preencheram os critérios de inclusão do estudo. Os animais apresentaram entre 2 e 20 anos de idade (média de 8,6 65 ,9 anos). O período de internação mínimo foi de 16 dias e o máximo de 263 dias (média de 104,2 $\pm 91,7$ dias).

As fêmeas representaram 55,6\% (5/9) dos casos, enquanto que os machos inteiros $44,4 \%$ (4/9) (Tabela 1). CRIPPS \& EUSTACE (1999a) não observaram variação na incidência da doença de acordo com o gênero. Na nossa rotina, não foram atendidos machos castrados, possivelmente devido ao alto custo do tratamento, tendo em contrapartida um prognóstico reservado com relação a desempenho atlético futuro. Quanto ao número de membros afetados, 22,2\% (2/9) apresentaram laminite nos quatro membros, enquanto $77,8 \%$ (7/9) apresentaram laminite apenas nos membros anteriores. Os membros torácicos são mais comumente afetados (provavelmente por suportarem mais peso do que os pélvicos), contudo, os membros pélvicos também podem estar envolvidos (geralmente em casos graves de sepse) (BELKNAP \& PARKS, 2011).
Tabela 1 - Perfil dos cavalos Crioulos internados com laminite crônica no período entre março de 2010 e outubro de 2011.

\begin{tabular}{lll}
\hline Sexo & Fêmeas & $55,5(5 / 9)$ \\
& Machos inteiros & $44,4(4 / 9)$ \\
& & \\
& Endotoxemia & $11,1(1 / 9)$ \\
Causa inicial & Resistência à insulina & $44,4(4 / 9)$ \\
& Desconhecida & $44,4(4 / 9)$ \\
Número de & 4 & \\
membros afetados & 2 & $22,2(2 / 9)$ \\
& & $77,8(7 / 9)$ \\
& & \\
Condições & Rotação $\geq 5^{\circ}$ & $66,7(6 / 9)$ \\
associadas & Afundamento $\geq 20 \mathrm{~mm}$ & $66,7(6 / 9)$ \\
& Afundamento unilateral & $11,1(1 / 9)$ \\
& medial da terceira falange & \\
\hline
\end{tabular}

Em 11,1\% dos cavalos, a laminite foi atribuída a endotoxemia (1/9), em 44,4\% (4/9) a SME e em 44,4\% (4/9) não foi possível identificar a causa primária, já que não apresentavam histórico de pleuropneumonia, aborto e cólica. Contudo, dos quatro animais com laminite de origem desconhecida, em apenas dois deles a SME foi excluída. Os outros dois não foram testados, não sendo possível descartar a presença da doença. A SME foi a causa predisponente mais comum de laminite neste estudo $(n=4)$, sendo descrita em cavalos adultos de todas as raças, com aparente maior incidência em pôneis. Ela se caracteriza por obesidade, hiperinsulinemia, devido à resistência à insulina, e predisposição à laminite. A laminite decorrente de SME pode variar em gravidade, no entanto, a maioria é de natureza crônica. Os achados radiológicos variam e não correspondem, necessariamente, ao grau da claudicação (HUNT \& WHARTON, 2010).

Apenas $\quad 11,1 \% \quad(1 / 9) \quad$ dos animais apresentaram afundamento distal unilateral medial da coluna óssea. A rotação ocorre com maior frequência que o deslocamento distal simétrico, que por sua vez é mais comum que o deslocamento distal unilateral. O deslocamento medial é mais comum, a superfície solar da falange distal no lado afetado se move distalmente no interior do casco. Esse tipo de deslocamento pode ser diagnosticado radiologicamente, usando a projeção dorsopalmar/ plantar horizontal da falange distal. Embora cada deslocamento possa ocorrer de forma independente, uma combinação dos três frequentemente ocorre nos cavalos (BAXTER, 2008).

Os pacientes foram monitorados diariamente por um médico veterinário e, 
dependendo da evolução no quadro clínico, os AINEs eram trocados ou até mesmo suspensos. Os antiinflamatórios se tornaram um componente central na farmacoterapia da laminite ao longo dos anos, pois reduzem a inflamação e a dor no interior do casco. A fenilbutazona $\left(4,4 \mathrm{mg} \cdot \mathrm{kg}^{-1}\right.$, a cada 12 horas) é um AINE potente no controle da dor, sendo o mais popular entre os clínicos (POLLIT, 2003; BELKNAP \& PARKS, 2011). Os principais AINEs disponíveis para uso clínico em equinos são os inibidores não seletivos de ciclooxigenase (COX) 1 e 2 flunixina meglumina, fenilbutazona, e cetoprofeno (BELKNAP \& PARKS, 2011). O tratamento sistêmico nos casos crônicos se baseia principalmente no controle da dor, que consiste na terapia predominantemente a base de fenilbutazona. A maior toxicidade da fenilbutazona em relação aos outros AINEs (a maioria dos relatos de ulceração no cólon dorsal direito estão associados ao uso de fenilbutazona) é devida a sua meia vida mais longa e por se acumular mais nos tecidos. Portanto, para evitar a toxicidade por ocasião de tratamento prolongado com fenilbutazona, recomenda-se suspender o seu uso por um período de 24 horas a cada 5-7 dias para permitir a depuração do fármaco do sistema (BELKNAP, 2006).

Ao avaliar o uso de AINEs, não se considerou o grau de claudicação, porque, quando a claudicação diminuía ou a fenilbutazona já vinha sendo usada há muito tempo o AINE era substituído por um de menor poder analgésico e menor toxicidade. Durante o período de internação, foi possível acompanhar alguns desses pacientes sem medicação com AINEs apresentando sinais sutis ou mesmo ausência de claudicação.

Os exames hematológicos e bioquímicos, repetidos periodicamente, não apresentaram variação em seus parâmetros atribuíveis à toxicidade resultante da administração contínua de AINEs, permitindo a sua utilização por períodos longos. Devido à potencial toxicidade gastrointestinal e renal causada pelos AINEs, muita atenção deve ser dada ao histórico do animal (isto é, sinais indicativos de úlceras gastro-intestinais ou doença renal), o estado geral do animal, a hidratação e realização de análises laboratoriais para avaliar a função renal (BELKNAP \& PARKS, 2011).

Dos animais avaliados, 66.7\% (6/9) apresentaram afundamento da terceira falange $\geq 20 \mathrm{~mm}$ em pelo menos um membro em pelo menos um estudo radiológico, contudo, nem todos apresentavam correlações clínicas correspondentes a afundamento da terceira falange, como a depressão da banda coronária. Curiosamente, o afundamento da coluna óssea não apresentou correlação com o grau de claudicação. BELKNAP \& PARKS (2011) sugerem que a distância de afundamento deve ser inferior a 18 a $20 \mathrm{~mm}$ para cavalos sadios de aproximadamente $450 \mathrm{~kg}$. O deslocamento distal simétrico ou sinking ocorre quando existe o colapso laminar uniformemente em toda a circunferência do casco e, dessa forma, a terceira falange afunda no interior do casco pela falta de sustentação (BAXTER, 2008).

Nossos resultados mostram que a ocorrência de afundamento não influenciou no prognóstico de sobrevivência dos animais, diferindo de ORSINI et al. (2010) que afirmam que a gravidade da claudicação é o indicador de prognóstico mais importante em cavalos hospitalizados com laminite, assim como a presença de deslocamento distal da terceira falange (afundamento) foi $o$ achado radiográfico mais significativo, associado a prognóstico desfavorável. KUMMER et al. (2006) observaram distâncias de afundamento maiores que CRIPPS \& EUSTACE (1999), atribuindo esse resultado a diferenças na posição dos marcadores. Diferenças na distância do afundamento (além do efeito da posição de marcadores) podem ser resultantes de variação na carga sustentada pelo membro, ou como consequência da laminite (KUMMER et al., 2006). Creditamos os altos índices de afundamento da coluna óssea à diferença na localização do marcador entre os diferentes estudos radiológicos. CRIPPS \& EUSTACE (1999b) demonstraram que diferentes operadores podem discordar quanto à colocação do marcador de metal na parede dorsal do casco e isso pode conduzir a diferenças na mensuração da distância de afundamento. Essas variações resultam da diferença de sensibilidade entre operadores na localização do limite dorsal da parede do casco.

Dos nove animais deste estudo, seis $(66,7 \%)$ apresentaram rotação $\geq 5^{\circ}$ em pelo menos um dos membros em pelo menos um estudo. A rotação palmar/plantar da falange distal em um ângulo superior a $5^{\circ}$ confirma o diagnóstico de laminite devido à rotação capsular (BELKNAP \& PARKS, 2011). Houve correlação $(P<0.05)$ entre o grau de rotação e o grau de claudicação. Ao contrário dos dados apresentados por STICK et al. (1982) e CRIPPS \& EUSTACE (1999a), nenhum dos nove pacientes retomou à carreira atlética, sendo todos encaminhados para reprodução. Em sua análise, STICK et al. (1982) avaliaram 91 casos de laminite e sugerem que cavalos com rotação $\leq 5,5^{\circ}$ possuem um prognóstico favorável quanto ao retorno à carreira atlética. Já animais com rotação $\geq 11,5^{\circ}$ possuem prognóstico desfavorável 
quanto a virem a desenvolver uma atividade esportiva, devendo ser mantidos na reprodução.

CRIPPS \& EUSTACE (1999a) analisaram os registros de 211 casos de laminite em cavalos e pôneis para determinar o significado clínico e radiológico de parâmetros como indicadores de prognóstico de laminite. Dos animais deste estudo, $77 \%$ voltaram à vida atlética; $3 \%$ não recuperaram totalmente a função atlética; e $20 \%$ morreram ou foram eutanasiados. EUSTACE \& CALDWELL (1989) demonstraram que animais com rotação da falange distal acima do limite sugerido de $11,5^{\circ}$ podem retornar a função atlética, se forem tratados com ferradura de coração e ressecção da parede dorsal. Já HUNT (1993) considera que quanto maior o grau de claudicação pior o prognóstico nos casos de laminite.

Apenas dois dos nove pacientes (22,2\%) foram submetidos à tenotomia do tendão flexor digital profundo (TFDP), mostrando estabilização da rotação no período pós-cirúrgico. MORRISON (2011) considerou 51\% de 245 tenotomias do TFDP bem sucedidas, ou seja, os animais sobreviveram pelo menos um ano após a cirurgia, mantendo boa condição corporal e claudicação inferior a grau 2 segundo Obel. A tenotomia do TFDP é um procedimento muito útil no tratamento de laminite crônica, sendo indicada quando há rotação progressiva, dor persistente, crescimento mínimo do casco, deformação da sola e contratura secundária do aparelho flexor (O'GRADY, 2011).

O casqueamento, baseado nos estudos radiológicos, tinha por objetivo corrigir a angulação da terceira falange com relação à parede do casco. Os objetivos gerais do tratamento buscam atender as alterações anatômicas e funcionais identificadas. O casqueamento é a base do tratamento de cavalos com rotação capsular/falangeana dorsal resultantes de laminite crônica. O objetivo imediato é realinhar a superfície solar do casco com a superfície solar da falange distal. Para fazer esse realinhamento com precisão, é necessária a orientação radiológica (O’GRADY, 2010). Após a estabilização do casco, era realizado o ferrageamento. O'GRADY (2010) relata maus resultados com ferrageamento em casos em que não houve estabilização do casco, tornando necessário o uso de anestesia local a fim de suspender o membro do cavalo com laminite para ferrageamento. Não há uma ferradura que seja eficaz no tratamento de todos os cavalos com rotação falangeana, no entanto, existem orientações que são úteis e podem ser adaptadas às necessidades de cada cavalo. As considerações principais envolvidas na seleção de ferraduras e técnicas de ferrageamento são reposicionar o ponto de quebra, fornecer suporte para a sola e ranilha e elevar os talões quando necessário (O’GRADY, 2010).

Mesmo sem retorno à carreira atlética, é importante frisar que nenhum dos animais tratados teve que ser eutanasiado e todos sobreviveram por período superior a seis meses após a alta. Consideramos o tratamento bem sucedido quando o animal permanece confortável com um mínimo de medicação, associado a casqueamento/ferrageamento corretivos periódicos. Segundo CRIPPS \& EUSTACE (1999a), o prognóstico para cavalos com laminite não difere do de pôneis, nos quais está incluído o nosso grupo de estudo. Apesar disso, STICK et al. (1982) observaram que pôneis apresentam rotação significativamente maior da falange distal do que cavalos.

\section{CONCLUSÃO}

A reabilitação de laminite crônica não segue um padrão, o tratamento é individual devendo ser adequado a cada caso. A resistência à insulina foi a causa predisponente a laminite mais comum neste estudo. O grau de claudicação pode ser usado como indicativo de rotação de terceira falange, visto a correlação que apresentam, contudo este não se correlaciona com a distância de afundamento. A terapia de suporte, cuidados intensivos de enfermagem, casqueamento e ferrageamento corretivos resultaram em alta taxa de sobrevivência com manutenção dos animais confortáveis sem uso de medicação após a alta.

\section{REFERÊNCIAS}

BAXTER, G.M. Review of support limb laminitis in horses. In: ANNUAL CONVENTION OF THE AMERICAN ASSOCIATION OF EQUINE PRACTITIONERS - AAEP, 54., 2008, San Diego, CA, USA. Proceedings... San Diego: AAEP, 2008. p.210-218. Disponível em: <http://www.ivis.org/proceedings/aaep/2008/ Baxter/chapter.asp. Online>. Acesso em: 07 jun. 2012.

BELKNAP, J.K. Treatment of the chronic laminitis case. In: NORTH AMERICAN VETERINARY CONFERENCE, 2006 Orlando, Florida. Proceedings... Orlando: NAVC, 2006. p.20; 78-80. Disponível em: <http://www.ivis.org/proceedings/ navc/2006/LA/031.asp?LA=1. Online $>$. Acesso em 07 jun. 2012 .

BELKNAP, J.K., PARKS, A. Lameness in the extremities: the foot. In: BAXTER, G.M. Adams \& Stashak's lameness in horses. 6.ed. UK: Wiley-Blackwell, 2011. Cap.5, p.536-556.

CRIPPS, P.J.; EUSTACE, R.A. Factors involved in the prognosis of equine laminitis in the UK. Equine Veterinary Journal, v.31, p.433-442, 1999a. 
CRIPPS, P.J.; EUSTACE, R.A. Radiological measurements from the feet of normal horses with relevance to laminitis. Equine Veterinary Journal, v.31, p.427-432, 1999 b.

EILER, H. et al. Physiologic assessment of blood glucose homeostasis via combined intravenous glucose and insulin testing in horses. American Journal of Veterinary Research, v.66, n.9, p.1598-604, 2005. Disponível em: <http://avmajournals.avma.org/ doi/abs/10.2460/ajvr.2005.66.1598>. Acesso em: 13 maio, 2013. doi: 10.2460/ajvr.2005.66.1598.

EUSTACE, R.A.; CALDWELL, M.N. Treatment of solar prolapse using the heart bar shoe and dorsal hoof wall resection technique. Equine Veterinary Journal, v.21, p.370-373, 1989. Disponível em: $<$ http://onlinelibrary.wiley.com/doi/10.1111/j.2042-3306.1989. tb02694.x/abstract>. Acesso em: 07 jun. 2012. doi: 10.1111/ j.2042-3306.1989.tb02694.x.

HOOD, D.M. Laminitis in the horse. Veterinary Clinics of North America: Equine Practice, v.15, p.287-294, 1999. Disponível em: <http://www.ncbi.nlm.nih.gov/pubmed/10472112>. Acesso em: 07 jun. 2012.

HUNT, R.J.; WHARTON, R.E. Clinical presentation, diagnosis, and prognosis of chronic laminitis in North America. Veterinary Clinics of North America: Equine Practice, v.26, p.141-153, 2010. Disponível em: <http://www.sciencedirect.com.ez47. periodicos.capes. gov.br/science/article/pii/S0749073909001023>. Acesso em: 07 jun. 2012. doi:10.1016/j.cveq.2009.12.006.

KUMMER, M. et al. The effect of hoof trimming on radiographic measurements of the front feet of normal Warmblood horses. Veterinary Journal, v.172, p.58-66, 2006. Disponível em: <http://www.sciencedirect.com.ez47.periodicos.capes.gov.br/ science/article/pii/S109002330500078X>. Acesso em: 07 jun. 2012. doi:10.1016/j.tvj1.2005.03.008.

MORRISON, S. Long-term prognosis using deep digital flexor tenotomy and realignment shoeing for treatment of chronic laminitis. Journal of Equine Veterinary Science, v.31, p.8996, 2011. Disponível em: <http://www.sciencedirect.com.ez47. periodicos.capes.gov.br/science/article/pii/S0737080610005526>. Acesso em: 07 jun. 2012. doi:10.1016/j.jevs.2010.12.008.

OBEL, N. Studies on the histopathology of acute laminitis. Uppsala, Sweden: Almquist and Wiksells Boktryckteri AK. 95p, 1948.

O'GRADY, S.E. Farriery for chronic laminitis. Veterinary Clinics of North America: Equine Practice, v.26, p.407-423, 2010. Disponível em: <http://www.sciencedirect.com.ez47.periodicos. capes.gov.br/science/article/pii/S074907391000043X>. Acesso em: 07 jun. 2012. doi:10.1016/j.cveq.2010.04.008.

O'GRADY, S.E. How to treat severe laminitis in an ambulatory setting. In: ANNUAL CONVENTION OF THE AMERICAN ASSOCIATION OF EQUINE PRACTITIONERS - AAEP, 57., 2011, San Antonio, Texas. Proceedings... San Antonio, 2011. p.270-279. Disponível em: <http://www.ivis.org/proceedings/ aaep/2011/270.pdf $>$. Online. Acesso em: 07 jun. 2012.

ORSINI, J.A. et al. Prognostic indicators of poor outcome in horses with laminitis at a tertiary care hospital. Canadian Veterinary Journal, v.51, p.623-628, 2010. Disponível em: <http://www. ncbi.nlm.nih.gov/pmc/articles/PMC2871359/>. Acesso em: 07 jun. 2012.

POLLITT, C.C. Laminitis. In: DYSON, S.J. Diagnosis and management of lameness in the horse. Missouri: Saunders, 2003. Cap.35, p.329-339.

STICK, J.A. et al. Pedal bone rotation as a prognostic sign in laminitis of horses. Journal of the American Veterinary Medical Association , v.180, n.3, p.251-253, 1982.

SWANSON, T.D. Clinical presentation, diagnosis, and prognosis of acute laminitis. Veterinary Clinics of North America: Equine Practice, v.15, p.311-319, 1999. 